\title{
Health Disparities Among African Americans
}

\author{
E. Kelly Sanford, PhD \\ Josie Scales, PhD \\ Essame Hamido, ED.D; \\ Waldine DeBerry, MSW
}

Tennessee State University

Doi: 10.19044/esj.2017.c1p2 URL:http://dx.doi.org/10.19044/esj.2017.c1p2

\begin{abstract}
The purpose of this research, "Health Disparities among African Americans," is to increase our understanding of the demographic differences of health disparities that exist in America. The state of Tennessee is ranked third in the nation for obesity, and 48th in the nation in education success (CDC, 2014). The correlation between these variables, along with the intervening variable of culture, suggests a strong impact on poor health and wellness among African Americans. This research suggests the peculiar nature of prejudice and its relationship to poor health, race, gender, social economic status and culture as it relates to precursors of poor health. This research assessed 300 TSU African American students on the comparative analysis of body weight and body muscle (BMI Ratio) to the national average of BMI Ratios for teens to young adults ranging in age from 17-20. Comparative analysis suggests that the participants were above the national BMI average. Active Learning Workshop was useful in helping participants to increase their understanding of the correlation between weight and health and wellness.
\end{abstract}

Keywords: Health Disparities, Race, Gender, Socioeconomics Status, Risk factors, Mortality and Morbidity

\section{Purpose}

The purpose of this research is to investigate causal factors related to the onset of increase weight and above the average body mass and body muscle by the index of TSU students ranging from 17-20 years of age.

\section{Aims of this Research are}

Aim 1: To increase understanding of the health disparities that existed among African Americans 
Aim 2: To identify how social factors such as: institutionalized racism, gender, socio-economic status (SES), and culture are related to health and wellness among African Americans

Aim 3: To investigate Body Weight to Body Muscle (BMI) Ratios are above the national average among TSU students ranging in age from 17-21 years old.

Aim 4: To inform of the importance of exercise and diet to health and wellness among African Americans.

\section{Why Is this Research Important?}

This research uses primary data to investigate the extent to which participants are above or below the national BMI and weight average. This has research significance because it allows an increased awareness of how social factors are related to health and wellness. The practical significance is for those understanding the correlation between exercising and diet can decrease the onset of poor health. Further, this is important since it increases our knowledge with the ability of changing our behaviors to improve health disparities and decrease mortality rates among African Americans in the United States of America.

\section{Literature Review}

It has been documented in research literature that social factors and Lifestyle Behaviors play an important role in the etiology of health and illness (Keil et al, 1984; and Vargas, 2000). Most epidemiological studies have failed to explain why these social factors and lifestyle behaviors affect health and illness among African Americans and other minority groups. In this paper, however, social factors such as race/ethnicity, socioeconomic status, and gender will be addressed in relation to health disparities.

\section{Race, Ethnicity, and Health}

Several studies suggested that differences exist in terms of race, gender, social class and health outcomes (National Center for Health Statistics, 2015; and Centers for Disease Control, 2015). It has been demonstrated in the research literature that African Americans tend to get sick at younger ages, and die sooner than European Americans (Williams et al, 2010). The Centers for Disease Control and Prevention (2013) stated that even European Americans who have low incomes and low levels of education tend to choose foods that are affordable, and junk foods and fast foods, yet they still have lower mortality rates compared to their African American counterparts in the similar socioeconomic status. African Americans having higher mortality rates may be due to prejudice and discrimination. According to Fischman 2010, stress related to prejudice and discrimination may increase blood pressure levels, as 
well as increase rates of strokes, cardiovascular and kidney diseases (Lewis et al. 2014). An explanation of the African American high rates of mortality suggested by Schnall and Kern (1986) is that they are forced to recognize the reality of their social situation, a reality in this country which is characterized by high unemployment, under employment, low wages and patterns of social discrimination based on race, all of which may lead to health disparities. Because regardless of African Americans social status, they are often faced with prejudice and discrimination on the jobs regardless of their qualifications. For example, they are first one to get laid off of a job when the job market turn down, they are last one on the job to get promoted and often get paid less when they are hired than their European American counterpart with the same educational attainment and job experience. African Americans are not able to get off work to seek preventive and primary health care. They are forced to recognize their social situation that they have no control over the matter if they want to survival in this society so they tend not to rebel. These social situations may lead to anxiety reaction and frustrations which lead to high levels of stress and in turn leads to chronic health and illness.

According to Kindig and Cheng (2013) the Hispanics who immigrated to the United States may be the healthiest and they tend to live in extended families which are very supportive and have community relationships which also build strong social support that may lead to better health outcomes. Nonetheless, low income and low education levels of minority groups are less likely to receive preventive services such as cancer screenings, and to seek medical treatment for chronic conditions such as high blood pressure, diabetes and other chronic diseases. They also tend to live in environments where they are exposed to toxic chemicals and other environmental hazards that may lead to other chronic diseases such as respiratory illness.

On the other hand, African Americans and European Americans with higher incomes and higher levels of education tend to have lower morbidity and mortality rates compared to their low income and low education counterparts. Regardless of income and education levels, European Americans live on the average three years longer than minority groups except Hispanic Americans. When investigating the relationship between race, income and infant mortality rates, Williams (2012) found that African American College Graduates are 2.5 times more likely to have higher rates of infant mortality than European American College Graduates. He maintained that the impact of race on health or may be independent of socioeconomic status, may be due to social and economic circumstances experienced over the course of life. He argued that the African American Graduates may have experienced adversity in their childhood that can affect their health outcomes in adulthood. Further study needs to be done to examine other social factors that may contribute to health outcomes of African Americans and other racial minorities. 


\section{Socioeconomic Status and Health}

Numerous studies revealed that socioeconomic status such as one's income level, education, and occupation influence one's health status in American Society (National Center for Disease Control, 2012). According to Cockerham (2013), low socioeconomic status is associated with less access to quality healthcare, increased likelihood of having an unhealthy lifestyle, and higher exposure to poor living conditions, injury, and disease. Other studies conducted by Farmer et al. (2010) maintained that people living in poverty are more likely to suffer from malnutrition, hazardous environmental conditions, housing and working conditions, and lack of clean water and sanitation.

Evidence in the research literature suggests that low socioeconomic status is associated with higher incidence and prevalence of chronic health problems and lower life expectancy (Bell et al, 2004). For example, a study of mortality rates conducted by Kindig and Cheng (2013) found that factors most strongly associated with higher mortality rates are those experienced by individuals who live below the poverty line and also those with no college education. They found that the rates of obesity are higher among people living below the poverty line. They stated that this is true because high-calorie processed food tends to be more affordable than fresh vegetables, fruits and lean meats or fish in their communities. Cockerham (2007) found in their study that lower income people are subjected to the most stress because they tend to have less coping mechanism. According to National Center for Health Statistics (2015), these individuals living below the poverty line are nearly ten times more likely to report that they suffer from psychological stress compared to those individuals with middle class status.

Another study by Hummer and Hernandez (2013) revealed that Americans who finished college live an average of ten more years than adults who did not finish school. They contend that higher educational attainment may be better linked to good health and good paying jobs, which can lead to better health care, a safer living environment, physically active recreation, and diet habits with more fresh foods. It was pointed out in their study that higher education tends to lead to greater knowledge about health issues, and individuals tend to make better health choices such as the choice to exercise, avoid smoking and heavy drinking, and to follow doctors' recommendations for managing health problems. National Institutes of Health has found that SES, more than race or ethnicity, predicts the likelihood of an individual's access to education, certain occupations, health insurance, and living conditions, including conditions where exposure to environmental toxins is most common, all of which are associated with the risk of developing chronic conditions. SES, in particular, appears to play a major role in influencing the prevalence of behavioral risk factors for cancer and other chronic conditions 
(for example, tobacco smoking, physical inactivity, obesity, and excessive alcohol intake, and health status). Further study is needed to address the overall impact of socioeconomic status on health outcomes.

\section{Gender and Health and Illness}

Previous research shows that there is a correlation between gender and health (Mohsenin et al 2009). According to the World Health Organization (2015), the average life expectancy in 2013 was 81 for women and 76 for men. Baker et al (2014) stated that the low life expectancy in males may be due to occupational hazards, dangerous sports, violence, and fast driving. It was pointed out that men are less likely than women to seek health care when they are ill, and when they see a doctor, men are less likely to disclose any symptoms of disease or illness that they may be experiencing (Cockerman, 2012).

Research studies suggested that women tend to care for others often without comprehension and they are more likely than men to suffer from stress and more likely than men to lack resources to pay for their own care, whereas, masculinity can encourage men to ignore pain or other symptoms of illness. As a result, they are less likely than women to consult a health care professional and get treatment. For the same reasons, males are more likely than females to engage in risky behaviors, such as smoking and drinking or extreme sports, that can affect their health outcomes. Studies show that African American females have higher rates of certain cancers (colon/rectal, pancreatic and stomach) than European American females. Among males, African American males have higher rates of certain cancers including prostate, lung/bronchus, colon/rectum and stomach. Freeman and Freeman (2013) revealed in findings regarding mental health that women are more likely than men to experience mental health issues.

\section{Methodology}

Three hundred male and female students, freshman through senior classification at Tennessee State University, were volunteer participants in a Health and Wellness project from the years of 2010-2015. Participants agreed to have their height, weight, and Body Mass Ratio (BMI) measured to assess and compare with national average BMI Ratios. Faculty monitored trained graduate students to make the assessments of student participants over the years. Equipment and technology were used to obtain proper weight, height, and BMI ratios. A health and wellness presentation was given to all participants, and a personal trainer offered to assist in proper diet and exercising to improve their BMI ratio ranging from cardio to light weight lifting. (While some students took advantage of the diet/exercise training it 
was not mandatory. However, information was disseminated with the theory that proper diet/exercise balance is important for health and wellness).

\section{Statistics}

Interval level data was collected to develop a matrix to compare students ranging from ages 17-22, freshman through senior classification. Their weight, height, and Body/Mass ratios were recorded. Comparison of the matrix was imposed on the National Average of BMI Ratio of similar age groups.

Comparative data analysis suggested that of the TSU female participants aged between17-20, their average weight was higher than the ideal average (Table 1).

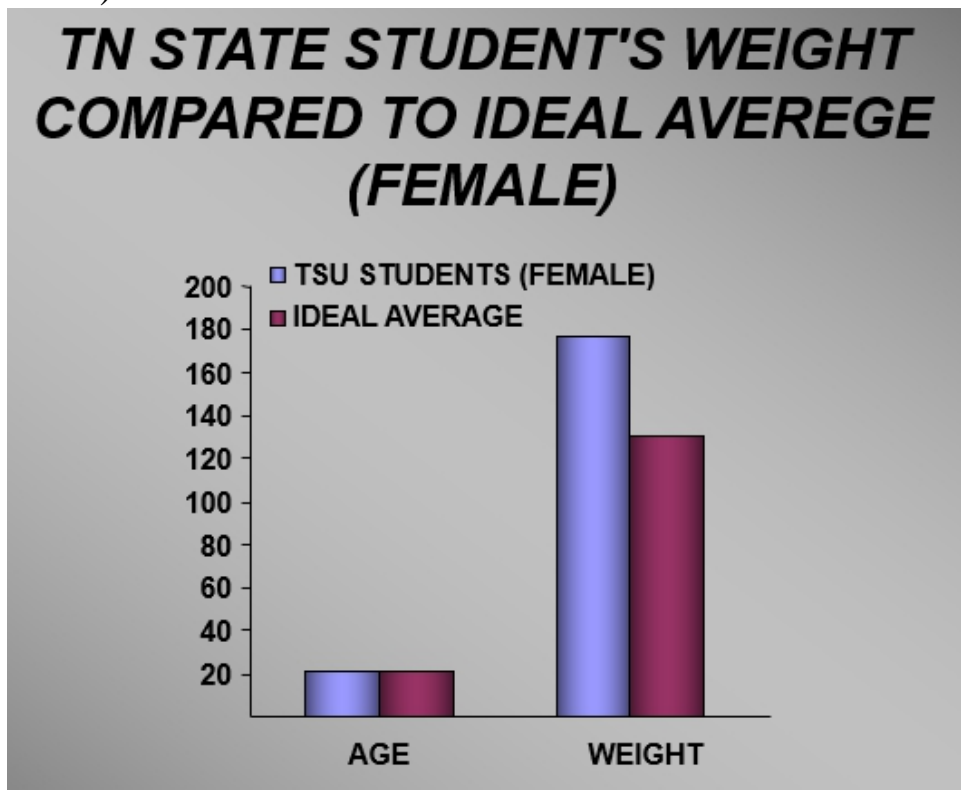

TABLE 1: TSU FEMALE STUDENT'S WEIGHT COMPARED TO IDEAL AVERAGE AGES 17-20

Accordingly, given the average range between 17-20 years old, the TSU female participants Body Mass was higher than the ideal average. Likewise, on the average, their Percent Body Fat was higher (Table 2). 


\section{TN STATE STUDENT'S WEIGHT INDEXES COMPARED TO IDEAL AVERAGE (FEMALE)}

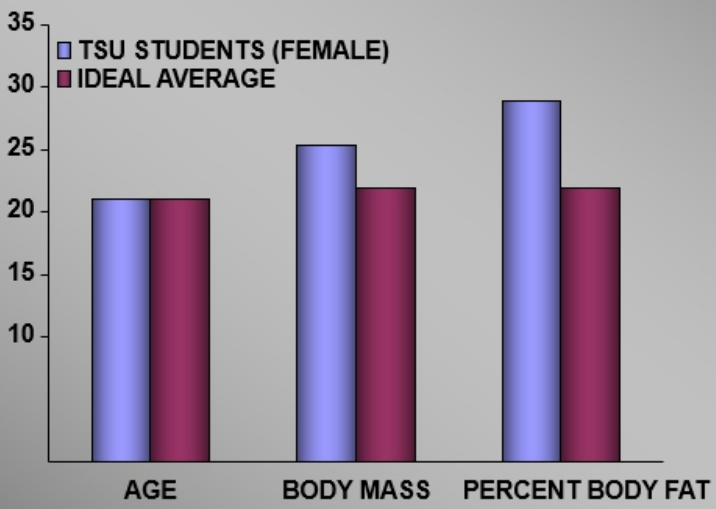

TABLE 2: TSU FEMALE STUDENT'S BMI RATIO COMPARED TO IDEAL AVERAGE BMI

AGE 17-20

Comparative analysis of the TSU male participants between the ages of 17-20, suggested that their weight was higher than the ideal average for males (Table 3).

\section{TN STATE STUDENT'S WEIGHT COMPARED TO IDEAL AVEREGE (MALE)}

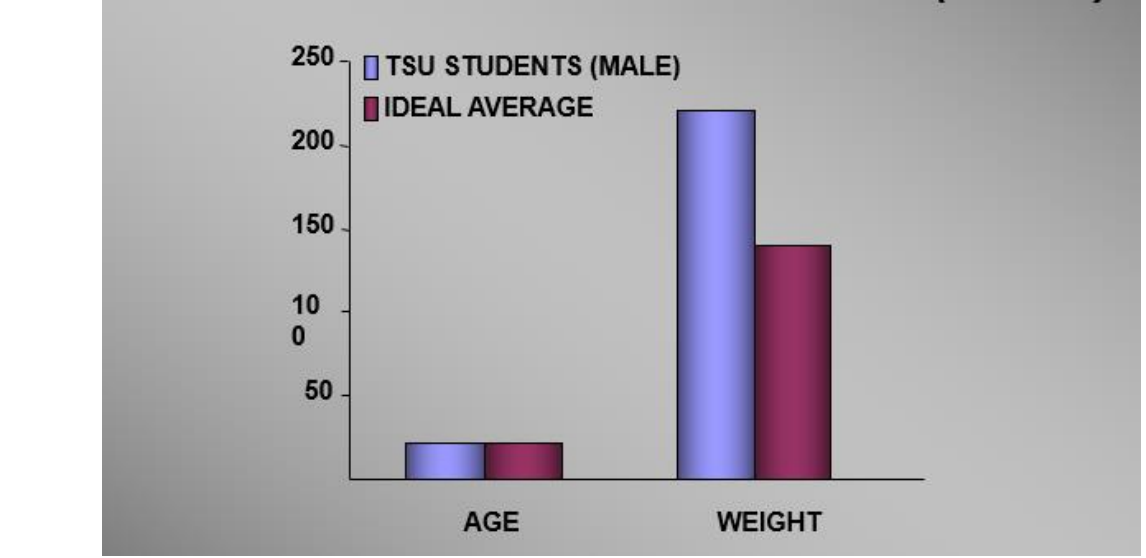

TABLE 3: TSU MALE STUDENT'S WEIGHT COMPARED TO IDEAL AVERAGE FOR MALES

AGE 17-20

BMI Ratio's for TSU male students was on the average higher than the comparative ideal average BMI for males of the same age range. 


\section{TN STATE STUDENT'S WEIGHT INDEXES COMPARED TO IDEAL AVERAGE (MALE)}

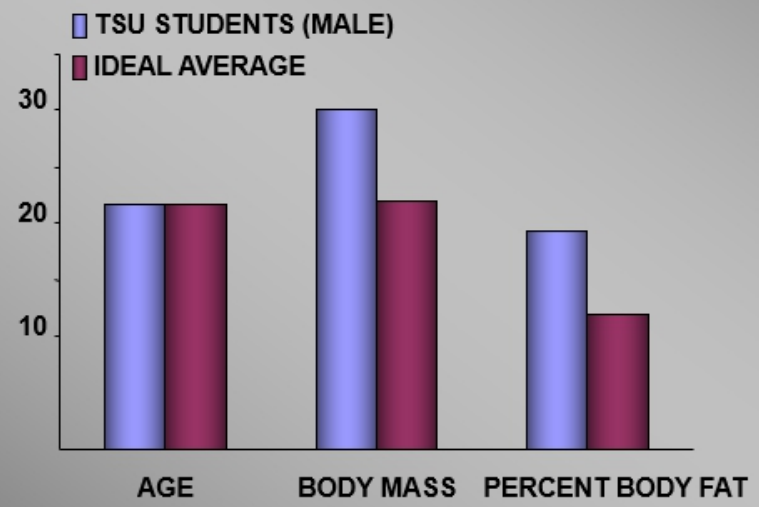

TABLE 4: TSU MALE STUDENT'S BMI COMPARED TO IDEAL BMI AVERAGE FOR MALES

AGE 17-20

\section{Results}

Comparative data analysis of 300 TSU males and females ranging in age from 17-20 years were on the average above the weight and BMI ratio of the Ideal Weight and BMI ratio. This data certainly had those TSU students that were below the ideal weight/BMI ratio and consisted of athletes, cheerleaders or the exceptional few in number of students that were within their desired weight/BMI ratios. However, the purpose of this research is to recognize the health disparities of African Americans in the United States. This comparative data analysis suggests the precursor or the beginning onset of how chronic illness starts is with being overweight and corresponding high BMI ratios. As indicated, social factors analysis on health has been undertaken mainly by social scientists who observed that biological factors alone cannot explain health behavior and illness. These social factors should be incorporated into health research so that social scientists can continue to investigate and understand the root causes of ill health and develop more effective disease prevention. Results from this data support the understanding that causal factors such as being overweight and having a high BMI ratio could contribute to early signs of the on-set of other causal factors leading to poor health and wellness. Youth certainly can cause people to think that their vital signs are normal. However, as life continues and the aging process continues, high blood pressure and hypertension may become the precursors to the on-set of other chronic illnesses. Participants attended the Active Learning 
Workshop afterward to increase their understanding of the contributing social factors leading to poor health and wellness.

This research supports the literature that social factors such as race, ethnicity, SES, gender, culture, and institutionalized racism (oppression and stress) are strong contributors to health disparities. The Center for Disease Control (CDC) national data indicates that African Americans are disproportionate in all chronic diseases and illnesses. This data suggest that teens to young adults are above the national average of weight and BMI ratios. Participants taking the workshop on improving health and wellness were able to assess their BMI ratio and begin using the simple diet exercise model to incrementally improve. Recognizing and understanding the social factors related as well as the correlation of being overweight and BMI location is a great beginning to prevention of poor health.

\section{Conclusion}

African Americans' health disparities indicate earlier deaths, decreased quality of life, loss of economic opportunities, and perceptions of injustice. For American society, these disparities translate into less productivity, higher health-care costs, and social inequity (Centers for Disease Control and Prevention (2013). Multiple factors may contribute to African American disparities, including socioeconomic factors (e.g., education, employment, and income), lifestyle behaviors (e.g., physical activity, dietary habits and alcohol intake), social environment (e.g., educational and economics opportunities, racial discrimination, and neighborhood and work conditions), and access to preventive health-care services (e.g., cancer, cardiovascular screening and vaccination) (William, 2012). This review demonstrates a need for further study to explain the African American differentials. However, the concept of "institutional racism" as an explanation of the African American/European American health outcomes gap merits further examination. The institutionalized process by which wages are set and labor is allocated requires further exploration.

\section{References:}

1. Baker, P. et al, 2014. “The Men's Health Gap: Men Must Be Global Health Equity Agenda." Bulletin of the World Health Organization 92: 618-670.

2. Centers of Disease Control and Prevention, 2014 (November 25). CDC Health Disparities and Inequalities Report-United States, 2014.

3. Centers for Disease Control and Prevention, 2013 (November 22). CDC Health Disparities and Inequalities Report-United States, 2013. MMWR 62 (3): all. 
4. Centers for Disease Control and Prevention. 2015. "Deaths: Final Data for 2013." National Vital Statistics Report 64(2).

5. CDC. Health, United States, 2004: table 30. Hyattsville, MD: US Department of Health and Human Services, CDC, National Center for Health Statistics; 2004. Available at http://www.cdc.gov/nchs/data/hus/hus04trend.pdf\#03.

6. CDC. Health, United States, 2004: table 53. Hyattsville, MD: US Department of Health and Human Service, CDC, National Center for Health Statistics; 2004. Available at http://www.cdc.gov/nchs/data/hus/tables/2004/04hus053.pdf.

7. Cockerham, W. C, 2007. Medical Sociology, $10^{\text {th }}$ Ed. Upper Saddle River, NJ: Prentice Hall.

8. Fischman, J., 2010. "The Pressure of Race." Chronicle of Higher Education, September 12.

9. Freeman, D. and Freeman, J, 2013. The Stressed Sex: Uncovering the Truth about Men and Women and Mental Health. Oxford: Oxford University Press.

10. House, James, 2002. Understanding Social Factors \& Inequalities in Health: $20^{\text {th }}$ Century Progress and $21^{\text {st }}$ Prospect. Journal of Health \& Social Behavioral, volume 43, No. 2.

11. Keil, J.E, 1993. Socioeconomics factors \& cardiovascular disease: a review of literature. Circulation, volume 88, Issue 4.

12. Kindig, D. and Cheng, E, 2013. "Even as Mortality Fell in Most U.S. Counties, Female Mortality Nonetheless Rose in 42.8 Percent of Counties from 1992 to 2006." Health Affairs 32 (3): 451-458.

13. Lewis, T. et al, 2014. "Self-Reported Experiences of Discrimination and Cardiovascular Disease." Current Cardiovascular Risk Reports 8 (1): 365-380.

14. National Center for Health Statistics. 2015. Health, United States, 2014. Hyattsville, MD: U.S. Government Printing Office.

15. National Center for Health Statistics, US, 2010. CDC Hyattsville, MD 563 6/2011. Available at: http://www.cdc.gov/data/hus/hus10.pdf.

16. National Center for Complementary, Alternative, or Integrative Health: What's in a Name?" Available at www.nami.org.

17. Ruiz, J. et al, 2013. "Hispanic Mortality Paradox: A Systematic Review and Meta-Analysis of the Longitudinal Literature." American Journal of Public Health 103 (3):e52-e60.

18. Schnall, P. L. and Kern, R, 1986. Hypertension in American Society: an introduction to historical materialist epidemiology. In: Conrad P, Kern R. eds. The Sociology of Health \& Illness. New York, N.Y.: St Martin Press; 73-89. 
19. USDHH, National Center for Health Statistics, 2004. Second National Health \& Nutrition Examination Survey 1999-2002

20. (NHANES II) Hyattsville, MD Center for Disease Control: 2000-2002, pages 1778.e, US Census Bureau, U.S. Bureau. US interim projections by age, sex, race, and Hispanic origin. Washington, DC: US Department of Commerce, US Census Bureau; 2004. Available at http://www.census.gov/ipc/www/usinterimproj.

21. US Department of Health and Human Services, (2010). Data 2010: Healthy people 2010 data base. Hyattsville, MD: US Department of Health and Human Services, CDC. National Center for Health Statistics: 2004. Available at http://wonder.cdc.gov.data2010/focus.htm.

22. Vargas, C. M. et al, 2000. Socio-demographic correlates of chronic health among adults: United States. Pain, 85 (1-2) 87-92.

23. Williams DR, Neighbors, N.W, Jackson, JS, 2003. Racial/ethnic discrimination and health: findings from community studies. American J Public Health 2003:93: 20-8.

24. Williams, D.R. et al, 2010. Race, Socioeconomics Status and Health: Complexities, Ongoing Challenges \& Research Opportunities. Annals of New York Acad Sci vol, 1186.

25. World Health Organization. 2015d. World Health Statistics 3015. Available at www.wo.int. 